\title{
Increased sharing of maternal HLA haplotypes among children exposed to diphenylhydantoin during pregnancy
}

\begin{abstract}
SUSAN E HODGE*, DON C VAN DYKE†, ALLEN S GOLDMAN FRAN HEIDE\&, ROBERT HILL\|, AND CHESTER M ZMIJEWSKIף

From *the Departments of Biomathematics and Psychiatry, UCLA School of Medicine, Los Angeles, California; tDivision of Developmental Disabilities, University Hospital School, Department of Pediatrics, University of Iowa Hospitals and Clinics, Iowa City, Iowa; $¥$ Center for Craniofacial Anomalies, University of Illinois School of Medicine, Chicago, Illinois; \$Division of Pediatrics and ||Department of Biostatistics, The City of Hope, National Medical Center, Duarte, California; and \Department of Pathology, Hospital of the University of Pennsylvania, Philadelphia, Pennsylvania, USA.
\end{abstract}

SUMMARY During investigation of HLA types among children exposed to diphenylhydantoin (DPH) in utero, we found no evidence of a distortion in haplotype sharing among affected sib pairs. Unexpectedly, however, we found a marked increase in the proportion of all sib pairs (not just affected ones) sharing maternal haplotypes. Among 14 two child families, 12 shared the maternal haplotype (expected would be seven); among families with more than two children the distortion was also pronounced. This finding, if verified in future studies, could indicate that something in the mothers, whether DPH use during pregnancy, or some genetic factor associated with seizures, or some effect of the seizures themselves, may be leading to non-random segregation of HLA haplotypes in their offspring.

Dilantin ${ }^{\circledR}$ (DPH, 5,5-diphenylhydantoin) was first introduced in 1938 and remains a first line anticonvulsant despite recent concerns regarding its use in pregnant women. ${ }^{2}$ Approximately 2 million persons in the United States take DPH regularly, with more than 6000 infants being estimated to have been exposed in utero to DPH annually in the United States. ${ }^{34}$ During the last 10 years, there has been increased concern regarding possible adverse effects of DPH on fetal morphogenesis. The specific syndrome recognised in its most extreme presentation has been called fetal hydantoin syndrome (FHS). ${ }^{5}$ It is characterised by four major categories of defects: craniofacial dysmorphism, prenatal onset of growth deficiency, central nervous system dysfunction with an IQ of less than 75, and an increased risk of major malformations. ${ }^{5}$ Only a percentage of those infants exposed, however, show adverse effects to DPH exposure. The reason for variations in susceptibility to DPH exposure is not known.

The incidence of major congenital malformations in the general population from study to study varies from 1 to $6 \%$ with a present consensus of approxi-

Received for publication 20 March 1987.

Revised version accepted for publication 5 October 1987 mately $3 \% .^{6}$ Children of women with seizures are believed to be at twice the risk of congenital malformations when compared to women without seizures. ${ }^{78}$ A study by Nakane et al ${ }^{9}$ found that the incidence of congenital malformations, when compared to women without seizures, was lowest when the mothers remained free of seizures and were not taking medication during the pregnancy, and highest in those where mothers were having seizures and taking medication. The group whose mothers had seizures but were not taking medication and the group whose mothers were without seizures and taking medication occupied an intermediate position. ${ }^{9}$

Studies in mice have demonstrated that susceptibility to DPH induced cleft palate is related to glucocorticoid receptor levels and is influenced by the $\mathrm{H}-2$ histocompatibility complex on chromosome $17 .^{10-12}$ The mechanism of susceptibility to environmental teratogens in animal models may in some cases be applicable to humans. The human evolutionary homologue of the mouse $\mathrm{H}-2$ complex is the HLA region on chromosome $6 .{ }^{13}{ }^{14}$ Thus, a study of HLA segregation in families of children exposed to DPH in utero may yield information about DPH susceptibility. 


\section{Methods and materials}

\section{THE FAMILIES}

We performed HLA typing on 18 families in which at least two children had been exposed to DPH in utero. All 50 of the children reported here were exposed to DPH in utero. For four of the 50 children DPH was taken during only a portion of the pregnancy, usually during the last trimester; the remaining 46 were exposed to DPH throughout the entire pregnancy (table 1). DPH dosages varied somewhat from mother to mother and sometimes from pregnancy to pregnancy. Moreover, some mothers were on additional anticonvulsant medication (for example, phenobarbitol, mysoline) as well as DPH. However, the common factor was that they all took DPH at levels required to control seizures, and that except for the four pregnancies mentioned above, they did so throughout their entire pregnancies (see Discussion section).

The 18 families were selected for HLA typing from a larger study of 65 families with DPH exposure during pregnancy. The 18 families who were HLA typed were selected because of willingness to participate and without regard to severity of symptoms or any other factors of which we are aware. Signed permission forms were obtained from family members before venepuncture. IRB approvals were obtained from the Children's Hospital of Philadelphia and the City of Hope, National Medical Center.

We identified a child as 'definitely affected' with FHS if he or she met all three of these criteria: (1) had been evaluated by a geneticist and given the diagnosis of FHS; (2) had nail hypoplasia on examination; and (3) had a negative history for alcohol or warfarin ${ }^{\circledR}$ exposure or the rare chromosomal or genetic syndromes associated with nail hypoplasia. If a child had minor malformations of FHS and no nail hypoplasia, he or she was classified as 'possibly affected'.

\section{TYPING FOR HLA}

Typing for the A, B, and C specificities of HLA was performed on peripheral blood lymphocytes obtained by standard venepuncture techniques using heparinised tubes. Typing was by the standard National Institutes of Health two stage microcytotoxicity method described by Mittal et al. ${ }^{15}$ A total of 110 antisera capable of detecting most of the recognisable HLA antigens was used. The sera were obtained from the NIH-NIAID Sera Bank, the collection of the Tissue Typing Laboratory of the University of Pennsylvania, and gifts from other investigators.

\section{HAPLOTYPE SHARING}

Two different analyses were performed: an affected sib pair analysis and a maternal haplotype analysis.

For the affected sib pair analysis, we determined whether the sharing of two, one, or zero haplotypes deviated significantly from the expected proportions of $0.25,0.50$, and $0 \cdot 25$. We looked at pairs of sibs who were both 'definitely affected'. We also did the analysis with the looser definition of being 'possibly affected'. Only full sibs were used here. We used a $\chi^{2}$ goodness of fit test.

For the maternal haplotype analysis, we determined whether pairs of sibs shared their maternal haplotypes more often than expected. We included maternal half sibs along with full sibs. This analysis

TABLE 1 The 18 families and their haplotypes.

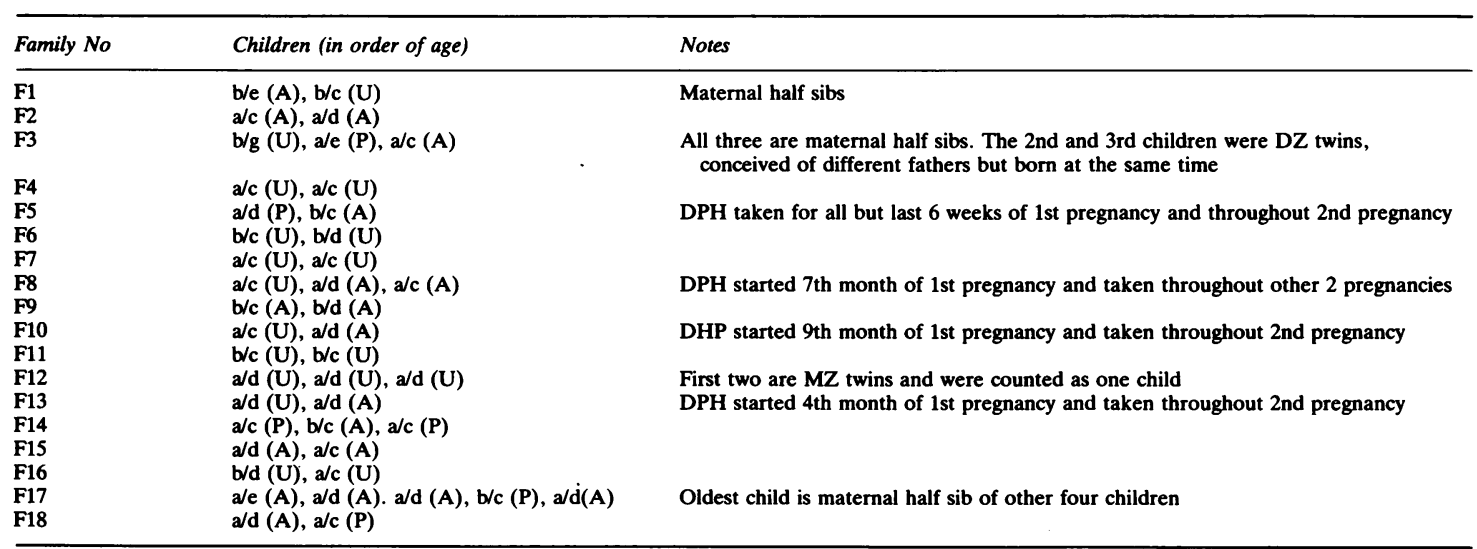

Mother is always $\mathbf{a} / \mathbf{b}$.

Father is always c/d (or e/f etc if more than one father).

$\mathbf{A}=$ definitely affected, $\mathrm{P}=$ possibly affected, $\mathrm{U}=$ unaffected. 
included both affected and unaffected children. The expected proportion of sib pairs sharing maternal haplotypes is one half.

To perform the maternal haplotype analysis in two child families, we simply counted each pair of children as either sharing or not sharing their maternal haplotypes, then tested the deviation from the expected proportion using an exact binomial test. In families with more than two children we analysed the data in two different ways. Method 1 selected the oldest child in each family and formed all pairs between that child and each subsequent child. Method 2 used all possible pairs in the family but weighted the proportions by appropriate factors. The deviation from expected was evaluated by a binomial test, either exact or using the normal approximation.

We give here a more detailed description of methods 1 and 2 for the maternal haplotype analysis. If a family has $s$ children, then a total of s(s1)/2 pairs can be formed among these children. However, the problem when $s>2$ is that all these s(s1)/2 pairs are not independent, either genetically or statistically. It can be shown, by reasoning analogous to that in reference 16 , that for this analysis of haplotype sharing from a single parent, the number of 'equivalent' sib pairs in an s child family is $\mathrm{s}-1$. In method 1 we selected the oldest child in the family and formed the s-1 pairs between that child and each subsequent sib. This has the advantage of being a straightforward statistical sampling procedure but the disadvantage of introducing a possible distortion in small samples. (For example, a three child sibship in which two children have maternal haplotype a and one has $b$ will be treated differently, depending on whether the $b$ child is the oldest or one of the a children is). In method 2 we formed all $s(\mathrm{~s}-1) / 2$ pairs and counted the numbers sharing or not sharing the maternal haplotypes, but we then multiplied these numbers by a factor of $2 / \mathrm{s}$ so that the family is weighted as if it had only s-1 pairs in it. For example, in the three child sibship described above, we count two pairs as sharing and one pair as not sharing; these counts 2 and 1 are then multiplied by the factor $2 / 3$, resulting in $4 / 3$ pairs sharing and $2 / 3$ pairs not sharing. This method has the disadvantage of using artificial fractional counts but the advantage of using all the pair sharing information contained in the sibship.

\section{Results}

Table 1 shows the family structures and HLA haplotypes. Three families (F1, F3, and F17) contained maternal half sibs. Family F12 had a pair of monozygotic (MZ) twins; the twins were treated as a single child for these analyses.
AFFECTED SIB PAIRS ANALYSIS

Using the stricter definition of 'definitely affected', we observed five affected sib pairs. When we added in three more 'possibly affected' pairs, we had a total of eight. Table 2 shows the haplotype sharing of these pairs, which gave no indication at all of deviating from the expected proportions of $1 / 4,1 / 2$, $1 / 4$.

\section{MATERNAL HAPLOTYPE ANALYSIS}

Table 3 summarises the sharing of maternal haplotypes in the 18 families. In the 14 two child families, 12 pairs shared their maternal haplotypes. If we analyse these two child families separately from the larger sibships, we obtain a $p$ value of 0.013 (two sided). (The exact binomial probability that 12 or more out of 14 pairs share maternal haplotypes, under the null hypothesis that the probability for each individual pair is $1 / 2$, is 0.0065 . Doubling this probability for a two sided test yields the $p$ value above.)

The remaining four families consisted of three with three children (F3, F8, F14) and one with five (F17). Method 1, forming pairs with the oldest child only, yields six pairs sharing maternal haplotypes and four pairs not sharing. When these figures are added to the 14 pairs from the two child families, the

TABLE 2 Haplotype sharing in affected sib pairs.

\begin{tabular}{lllll}
\hline & \multicolumn{5}{l}{ No of haplotypes shared } \\
\cline { 2 - 5 } & 2 & 1 & 0 & Total \\
\hline Definitely affected & 1 & 4 & 0 & 5 \\
Definitely affected+possibly affected & 1 & 6 & 1 & 8 \\
\hline
\end{tabular}

TABLE 3 Sharing of maternal haplotypes in the 18 families.

\begin{tabular}{|c|c|c|c|}
\hline Family & $\begin{array}{l}\text { No of children } \\
\text { in family }\end{array}$ & $\begin{array}{l}\text { No of children } \\
\text { sharing maternal } \\
\text { haplotype }\end{array}$ & Shared haplotype \\
\hline F1 & 2 & 2 & A11, B22 \\
\hline F2 & 2 & 2 & A1. B8 \\
\hline F3 & 3 & 2 & A11, Bw35 \\
\hline F4 & 2 & 2 & A3, B14 \\
\hline F5 & 2 & None & - \\
\hline F6 & 2 & 2 & A26, B17 \\
\hline $\mathbf{F} 7$ & 2 & 2 & A1, Bw38 (16) \\
\hline F8 & 3 & 3 & $\mathrm{~A} 2, \mathrm{~B} 40$ \\
\hline F9 & 2 & 2 & A30, B40 \\
\hline F10 & 2 & 2 & A9. B7 \\
\hline F11 & 2 & 2 & Aw34, B13 \\
\hline F12 & 2 & 2 & A2, B18 \\
\hline F13 & 2 & 2 & A3, Bw44 (12) \\
\hline F14 & 3 & 2 & A2, B15, Bw6 \\
\hline F15 & 2 & 2 & A2, B27 \\
\hline F16 & 2 & None & - \\
\hline F17 & 5 & 4 & A1, B8 \\
\hline F18 & 2 & 2 & $\mathrm{~A} 2, \mathrm{~B} 40$ \\
\hline
\end{tabular}


TABLE 4 Analysis of maternal haplotype sharing.

\begin{tabular}{llll}
\hline & \multicolumn{2}{l}{ No of pairs } & \\
\cline { 2 - 4 } & Share & Do not share & Total \\
\hline $\begin{array}{c}\text { Two child families } \\
\mathrm{p}=0.013 \text { (2 sided) }\end{array}$ & 12 & 2 & 14 \\
$\begin{array}{c}\text { All families } \\
\text { Method 1 } \\
\mathrm{p}=0.023 \text { (2 sided) }\end{array}$ & 18 & 6 & \\
$\begin{array}{c}\text { Method 2 } \\
\mathrm{p} \cong 0.03 \text { (2 sided) }\end{array}$ & 17.7 & 6.3 & 24 \\
\hline
\end{tabular}

total (18 out of 24 pairs sharing maternal haplotypes) deviates significantly from expected, with $\mathrm{p}=0.023$ (two sided). Method 2 yields similar results: $5 \cdot 7$ 'pairs' share maternal haplotypes and $4 \cdot 3$ do not. The $p$ value, using the normal approximation to the binomial distribution, is approximately 0.03 . Since both methods yield such similar results, we simply use a $p$ value of 0.03 in the ensuing discussion. This $p$ value is for the whole data set, including both the two child families and the families with more than two children. Table 4 summarises these results.

\section{Discussion}

We found no evidence for a distortion in the sharing of HLA haplotypes in affected sib pairs, either 'definitely affected' or 'possibly affected'. However, we observed a striking and quite unanticipated increase in the sharing of maternal haplotypes among all sib pairs, whether affected or not. This distortion was statistically significant.

We urge caution in interpreting this increase in maternal haplotype sharing, especially since this finding does not represent the initial hypothesis of interest but was only noticed serendipitously as we examined the data. Certainly our finding needs to be replicated in independent work before it can be accepted as proven.

When the increased sharing of maternal haplotypes was noted, we went back and broke the families down into affected sib pairs (that is, both sibs affected), unaffected pairs (both sibs unaffected), and mixed pairs (one sib affected, one unaffected). We wanted to see whether any of these subgroups exhibited either more or less sharing of maternal haplotypes than the other groups. However, there was no evidence for this, that is, the distortion in maternal haplotype sharing was about the same in all three groups.

Another important question, if this finding holds up under further study, is whether any particular
HLA allele(s) or haplotype(s) are associated with this maternal sharing. In our sample, there is no evidence for preponderance of any HLA-A or -B allele or any A-B haplotype. For example, the most prevalent $A$ antigen in our sample is $A 2$; this is also the most frequent in North American Caucasians, with a gene (allele) frequency of about $27 \% .{ }^{17}$ However, these observations do not rule out the possibility of an association. For one thing, our sample is too small to detect any but the most striking association. For another, we have data only on the A and B specificities. An association could exist with, for example, the alleles at the DR locus but not show up strongly with the $A$ and $B$ alleles.

We also reanalysed the data, excluding the four children (one each in families F5, F8, F10, and F13) for whom DPH was taken during only part of the pregnancy. This left 12 two child families, of which 11 pairs shared the maternal haplotype. The two sided $p$ value is 0.006 for the two child families considered alone. When the three larger families (F3, F14, and F17) are included, the sharing is still significant at the 0.05 level $(\mathrm{p}=0.04$ using method 1 , $\mathrm{p}=0.05$ using method 2).

Throughout this study we report two sided $p$ values, rather than one sided ones, since we had not specified a priori that we would examine maternal as opposed to paternal haplotype sharing. No doubt, if we had observed such a striking increase in paternal haplotype sharing instead, we would still have reported it. Therefore, a two tailed test, being more conservative, seemed appropriate.

We do not know why there should be increased sharing of maternal HLA haplotype in the offspring of these mothers. One explanation would be to attribute this to segregation distortion. However, previous reports in humans and mice have noted distortions of segregation ratios in males but not in females. ${ }^{18}{ }^{19}$ Klitz et al ${ }^{19}$ concluded that there is no significant segregation distortion in humans.

The phenomenon seen in these families does seem to depend on the mother and not on whether the child has FHS. It also appears that a genetic factor is involved which is linked to HLA on chromosome 6. However, we cannot tell whether the increased haplotype sharing results from the action of DPH on meiosis or from some underlying genetic factor predisposing the woman to have seizures, or even possibly from the effects of the seizures themselves. In addition, we do not know whether the increased sharing results from segregation distortion at the genetic level or from some selective effect either on maternal germ cells or on the conceptus after fertilisation.

As we pointed out above, this finding needs to be confirmed independently. We hope that this report 
will stimulate other investigators to examine this question in their data. If the phenomenon of maternal haplotype sharing which we have observed here is replicated in future studies, it may help shed light on the genetics of seizures and FHS.

This work was supported in part by the following grants: DE-04622, DE-05041 (to ASG), and DK31813 (to SEH) from NIH, a Thrasher Foundation Grant to ASG, a City of Hope, National Medical Center grant (SO7RR05471), and a Ruth Gelb Research Fellowship to DVD, and an NIH Research Career Development Award DK-01145 to SEH. We thank M Wilkey, G McAllister, J Kearns, G Ducker, J Bailey, L Sojka, and J Soucek for technical assistance. We are grateful to Drs G L Klein, Alison A Fox, B H Athreya, J M Miller, J Newman, J W Hanson, and David A Greenberg for critical review of this manuscript. We are most grateful to The Spokesman, the National Epilepsy Foundation newsletter, to Dr Henry S Cecil and the staff of child development of the Children's Hospital of Philadelphia, to the staff of the Children's Seashore House, and to the staff of the San Gabriel/Pomona Regional Center for their support. We are particularly grateful to the families who participated in this study and to the assistance and cooperation of the editorial staff of The Spokesman.

\section{References}

1 Merritt HKH, Putman TJ. Sodium dipenylhydantoinate in the treatment of convulsive disorders. JAMA 1938;111:1068-73.

2 Penry JK, Newmark ME. The use of antiepileptic drugs. Ann Intern Med 1979;89:207-18.

3 DHEW Publication No 78-5217. The dental implications of epilepsy. Rockville, MD: Health Services Administration, 1977.

${ }^{4}$ Hanson JW, Myrianthopoulos NC, Harvey SMA, Smith DW. Risks to the offspring of women treated with hydantoin anticonvulsants with emphasis on the fetal hydantoin syndrome. J Pediatr 1976;89:662-8.
${ }^{5}$ Hanson JW, Smith DW. The fetal hydantoin syndrome. $J$ Pediatr 1976;87:285-90.

${ }^{6}$ Kalter H, Warkany J. Congenital malformations-etiologic factors and their role in prevention. $N$ Engl J Med 1983;808: 424-31, 491-7.

${ }^{7}$ Nelson KB, Ellenberg JH. Maternal seizure disorder, outcome of pregnancy and neurological abnormalities in the children. Neurology (Minneap) 1982;32:1247-54.

${ }^{8}$ Fedrick J. Epilepsy and pregnancy: a report from the Oxford Record Linkage Study. Br Med J 1983;ii:442-8.

9 Nakane Y, Okuma T, Ryo T, et al. Multi-institutional study on the teratogenicity and fetal toxicity of antiepileptic drugs: a report of a collaborative group in Japan. Epilepsia 1980;21: 663-80.

10 Goldman AS, Baker MK, Gasser DL. Susceptibility to phenytoin-induced cleft palate in mice is influenced by genes linked to $H-2$ and $H-3$. Immunogenetics $1983 ; 18: 17-22$.

11 Gupta C, Katsumata M, Goldman AS. $H-2$ influences phenytoin binding and inhibition of prostaglandin synthesis. Immunogenetics 1984;20:667-76.

12 Gupta C, Goldman AS. Dexamethasone-induced phospholipase A2-inhibitory proteins (PLIP) influenced by the $\mathrm{H}$-2 histocompatibility region (41980). Proc Soc Exp Biol Med 1985;178: 29-35.

13 Ivanyi P. Some aspect of the $H-2$ system, the major histocompatibility system in the mouse. Proc $R$ Soc Lond 1978;202:117-58.

14 Lamm LU, Friedrick U, Petersen GH, et al. Assignment of the major histocompatibility complex to chromosome 6 in a family with a epicentric inversion. Hum Hered 1974;24:273-84.

15 Mittal KK, Mickey MR, Singal DP, Terasaki PE. Serotyping for homo transplantation XVIII refinement of microdroplet lymphocyte toxicity test. Transplantation 1968;6:913-27.

16 Hodge SE. The information contained in multiple sibling pairs. Genet Epidemiol 1984;1:109-22.

17 Terasaki.PI, Tiwari JL. HLA and disease associations. Heidelberg: Springer-Verlag, 1984:8-10.

18 Khoury MJ, Erickson JD, Levy MJ. Paternal effects on the human sex ratio at birth: evidence from interracial crosses. $A m \mathrm{~J}$ Hum Genet 1984;36:1103-11.

${ }^{19}$ Klitz W, Lo SK, Neugebauer M, Baur MP, Albert ED, Thomson G. A comprehensive search for segregation distortion in HLA. Hum Immunol 1987;18:163-80.

Correspondence and requests for reprints to Dr D C Van Dyke, Division of Developmental Disabilities, Department of Pediatrics, 209 University Hospital School, Iowa City, Iowa 52242, USA. 